\title{
Omitted Pulmonary Arteriovenous Fistula Complicated With Brain Abscess
}

\author{
Shengxun $\mathrm{Lu}$
}

\begin{abstract}
A 52-year-old female, diagnosed with a bilateral lower lobe pulmonary arteriovenous fistula (PAVF) at the age of 14 due to dyspnea, underwent a lobectomy of the right lower lung with a severe lesion, and symptom relief without treatment of the minor lesion in the other lung. She had active chest tightness since 42 years old and headaches 10 days before hospitalized, was diagnosed with PAVF and brain abscess, and cured by a successful PAVF embolization and a surgical resection of brain abscess. In the same patient, both methods of treatment for PAVF were all satisfactory, and each has advantages and indications.
\end{abstract}

Keywords: Pulmonary arteriovenous fistula; Brain abscess; Transcatheter embolization

\section{Introduction}

Pulmonary arteriovenous fistula (PAVF) is a congenital or acquired pulmonary vascular malformation (PVM) with a thinwall and dilated structure. PAVF brings about anatomic rightto-left shunts between pulmonary arteries and veins without capillary beds for gas exchange and filtration. The patients with serious shunt experience chest tightness, dyspnea, palpitation, thoracalgia, hemoptysis and other symptoms. PAVF predisposes to complication of hemorrhage, paradoxical embolization leading to stroke and brain abscess $[1,2]$.

\section{Case Report}

A 52-year-old woman presented to neurology department with a chief complaint of headache for 10 days, and had an anamnesis of surgical therapy for right lower lobe PAVF before 38 years. She was hospitalized with active chest tightness before 10 years and diagnosed with left lower lobe PAVF. The lesion was significantly more severe than the original. Family his-

Manuscript accepted for publication March 20, 2017

Department of Cardiovascular Surgery, The 150th Central Hospital of PLA, Luoyang 471031, China. Email: lushengxun2008@126.com

doi: https://doi.org/10.14740/jmc2798w tory included mother dying of PAVF complicated with severe emptysis, and daughter suffering from PAVF in left lower lobe. Outpatient computed tomography (CT) displayed occupying lesion in the occipital lobe of the right brain and peripheral edema (Fig. 1a), multiple nodules in the left lower lung and vascular malformations with high probability (Fig. 1b, c). On admission, body temperature was $36.2^{\circ} \mathrm{C}$, cyanotic lips, foreheads visible vascular nevus and limbs tendon hyperreflexia. Blood examination showed a white blood cell count of 9,030 cells $/ \mu \mathrm{L}$, neutrophil ratio of $79 \%$, hemoglobin of 151 $\mathrm{g} / \mathrm{L}$, hematocrit of $47.9 \%$ and hypersensitive $\mathrm{C}$-reactive protein of $14.3 \mathrm{mg} / \mathrm{L}$. Arterial blood gas (ABG) reported resting oxygen partial pressure of $58.0 \mathrm{~mm} \mathrm{Hg}$ and oxygen saturation of $83 \%$. Rachiocentesis results showed intracranial pressure was $130 \mathrm{~mm} \mathrm{H}_{2} \mathrm{O}$, cerebrospinal fluid was colorless transparent (protein, $1.862 \mathrm{mg} / \mathrm{dL}$; chloride, $117.5 \mathrm{mmol} / \mathrm{L}$; glucose, $2.50 \mathrm{mmol} / \mathrm{L}$ ), Paneth test was weakly positive, red blood cell counts was 10 cells $/ \mu \mathrm{L}$ and leucocyte count was 335 cells $/ \mu \mathrm{L}$. Pulmonary computed tomography angiography (CTA) presented left lower lobe PVM and PAVF, and the lesion diameter was about $10.5 \mathrm{~mm}$ (Fig. 2a). Head MRI reported that brain abscess occurred in the right occipital lobes, and there were many of old cerebral infarctions (Fig. 2b).

As antibiotic therapy, $3.0 \mathrm{~g} /$ day of ceftazidine and $1.6 \mathrm{~g} /$ day of norvancomycin hydrochloride had been administered intravenously. The oxygen saturation was increased to $94 \%$ from $83 \%$ by supplementary oxygen. Headache symptom in the patient improved significantly, and interventional embolization took place under local anesthesia after 7 days (Fig. 3). Spring coils were implanted to the aneurysm neck, and angiography showed that the fistula was closed completely. Chest tightness disappeared immediately after occlusion. Postprocedure $\mathrm{ABG}$ indicated that resting oxygen partial pressure increased to $89.0 \mathrm{~mm} \mathrm{Hg}$ and oxygen saturation increased to $99 \%$. Excision of cerebral abscess was performed 5 days later, and a cystic lesion and brown turbid pus were shown. Pathological findings suggested chronic granulomatous disease and acute inflammation. Twelve days after the operation, the patient was followed up for MRI: the area of the right occipital lobe lesion was blurred, and the white matter could be seen with edema band. She was discharged on the 22nd day after operation.

\section{Discussion}

PAVF was first reported by Churton in 1897 [3]. Due to the 

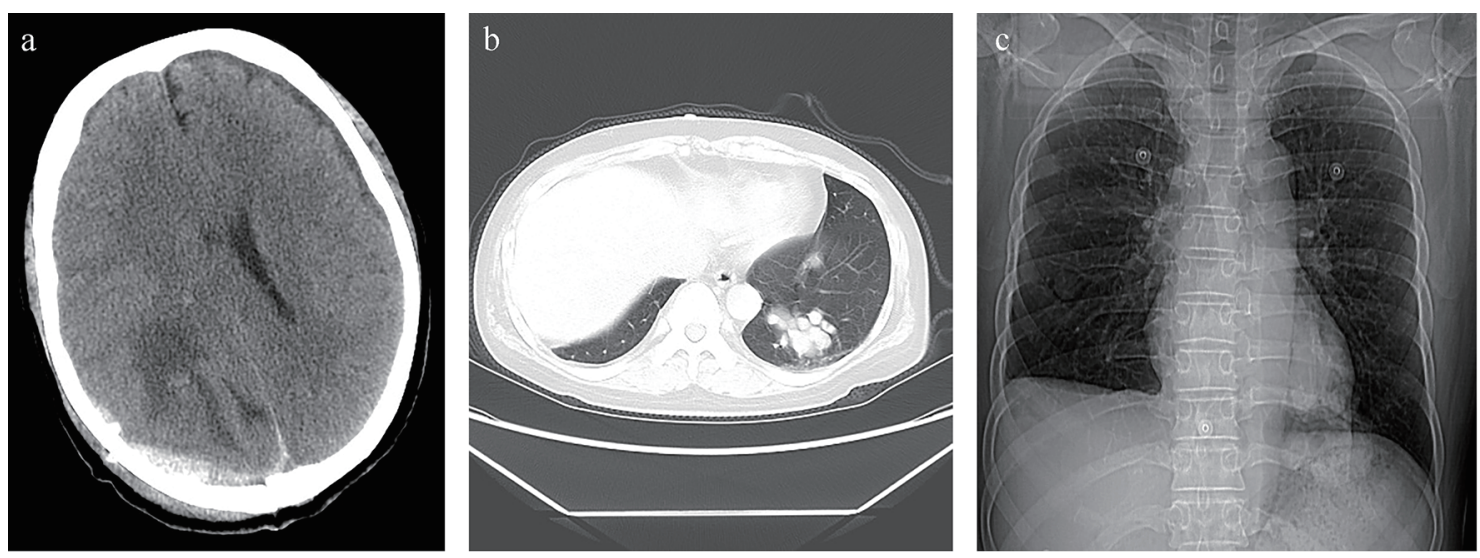

Figure 1. (a) Head CT showing an irregular low density in the right occipital lobe. (b) Chest CT scan illustrating multiple nodules with varying sizes and uniform density in the left lower lobe. (c) Chest roentgenogram shows PAVFs occurred in the left lower lobe and the residual lobes had a good compensation in right lung after operation.

limitations of imaging, diagnosis of such diseases was more difficult, and was considered to be a rare disease before 1950s. Hereditary hemorrhagic telangiectasia (HHT) is the most common cause of PAVFs in patients, an autosomal dominant disease, also known as Randu-Osler-Weber disease [4, 5]. A diagnosis mainstay of HHT is the Curacao criteria, which represent an international consensus initially published in 2000 . A diagnosis of definite HHT is recognized if at least three of the following are present: 1) epistaxis (spontaneous, recurrent); 2) telangiectasia (multiple at characteristic sites, i.e., lips, oral cavity, fingers, nose); 3) visceral lesions, including, but not limited to, GI telangiectasia (with or without bleeding), pulmonary, hepatic, cerebral, and spinal arteriovenous malformations; and 4) family history of a first-degree relative with HHT according to these criteria [6]. In addition, mutations in three genes (ENG, ACVLI/ALK1 and SMAD4) with varying clinical phenotypes have been identified. The genes can also be used to diagnose HHT [3]. At present, the main physical exam in clinical diagnosis of PAVF is CTA, pulmonary artery angiography, contrast echocardiography and MRI [7]. The most effective treatment is surgical excision and interventional embolization, and the latter as a gold standard in the treatment of PAVF is prevalent.

No matter whether there is a symptom or not, embolization should be performed for PAVF with a diameter greater than $3 \mathrm{~mm}$, which can effectively reduce the risk of complications such as paradoxical embolism, namely "3 mm rule" [8]. Surgical treatment as a supplementary approach is mainly used in patients with interventional treatment failure or who need lung transplantation [3]. It should be noted that PAVF often increased in size and number during pregnancy, and a small proportion of women experienced life-threatening complications $[1,9]$.
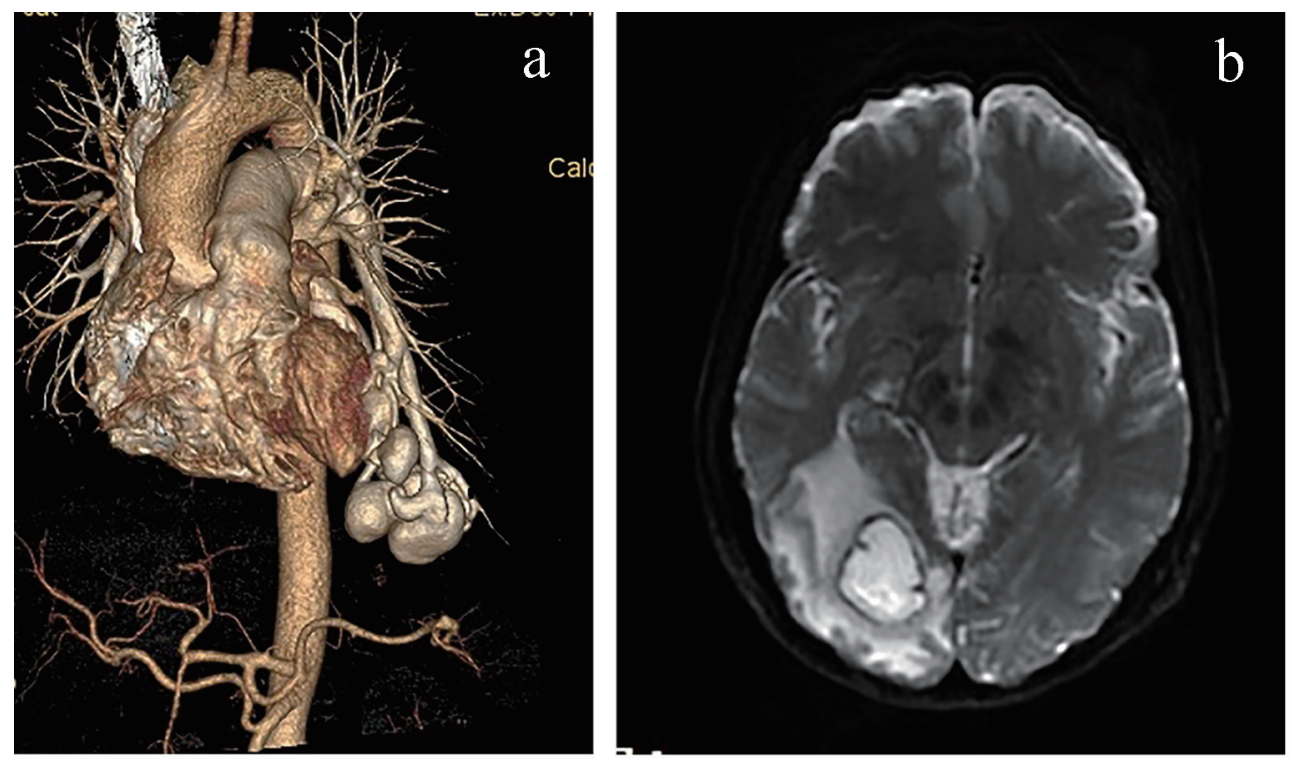

Figure 2. (a) Three-dimensional CT imaging displaying pulmonary arteriovenous malformation, tortuosity and dilation in left lower lobe (arrow). (b) Cranial MRI showing a cystic lesion with peripheral edema in the right occipital lobe, brain abscess formation. 

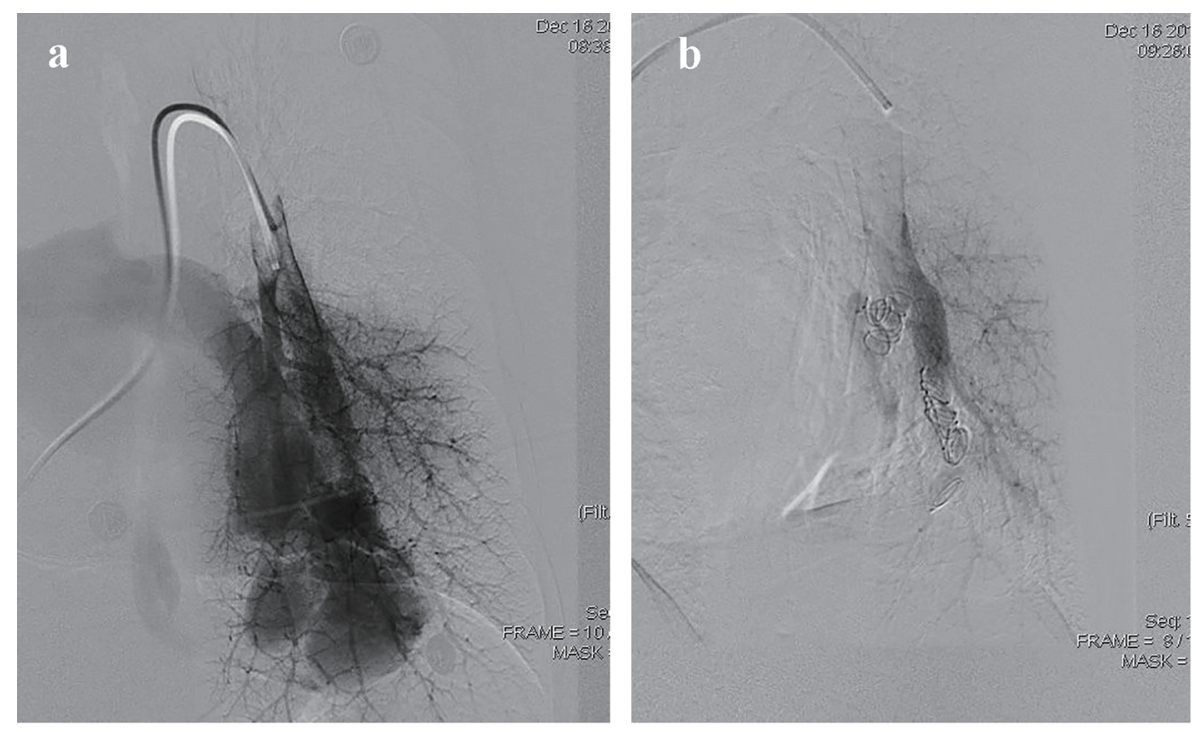

Figure 3. (a) Pulmonary angiography shows pulmonary blood flow into pulmonary veins via PAVF directly. (b) The PAVF shunt disappeared after coil embolization.

The patient was diagnosed with bilateral lower lobe PAVF at the age of 14, and received lobectomy, and the mild lesion in the other lung was without treatment. With the progression of the lesion, the symptom reappeared before 10 years, complicated with multiple cerebral infarction and brain abscess, and complete treatment with interventional embolization. In one case, two different treatment methods were used successively, and good therapeutic effect was achieved. Lobectomy is a radical treatment. The residual lobes have a good compensation after operation in Figure 1c, but the disadvantage is trauma. Advantages of transcutaneous catheter embolization are minimally invasive and rapid recovery. The defect is that the lesion may continue to grow without excision, and there are potential hazards of occlude abscission, bleeding, stroke, and other risks.

The case gives us a revelation that PAVF should be diagnosed and treated early to avoid infarct, hemorrhage, brain abscess and other complications. Young patients with the single lesion, especially the women who have a pregnancy plan should be suggested to apply a radical surgical treatment. Geriatric patients and those with multiple lesions should receive embolization treatment aim to improve symptoms with minimally invasive [10].

\section{Disclosure}

No potential conflicts of interest relevant to this article were reported. Ethical approval was given by the medical ethics review committee of hospital.

\section{References}

1. Faughnan ME, Palda VA, Garcia-Tsao G, Geisthoff
UW, McDonald J, Proctor DD, Spears J, et al. International guidelines for the diagnosis and management of hereditary haemorrhagic telangiectasia. J Med Genet. 2011;48(2):73-87.

2. Shovlin CL. Pulmonary arteriovenous malformations. Am J Respir Crit Care Med. 2014;190(11):1217-1228.

3. Churton $T$. Multiple aneurysm of pulmonary artery. BMJ. 1897;1:1223.

4. McDonald J, Bayrak-Toydemir P, Pyeritz RE. Hereditary hemorrhagic telangiectasia: an overview of diagnosis, management, and pathogenesis. Genet Med. 2011;13(7):607-616.

5. Mehrbod N, Chitsaz A, Saadatnia M, Manouchehri N. Stroke in a patient with pulmonary arteriovenous fistula: A case report study. Adv Biomed Res. 2013;2:84.

6. Shovlin CL, Guttmacher AE, Buscarini E, Faughnan ME, Hyland RH, Westermann CJ, Kjeldsen AD, et al. Diagnostic criteria for hereditary hemorrhagic telangiectasia (Rendu-Osler-Weber syndrome). Am J Med Genet. 2000;91(1):66-67.

7. Fan C, Zhou Z, Yin N, Yang J. Detection of pulmonary arteriovenous fistula with three-dimensional computed tomographic angiography. Eur Heart J. 2016;37(42):3210.

8. Pollak JS, Saluja S, Thabet A, Henderson KJ, Denbow N, White RI, Jr. Clinical and anatomic outcomes after embolotherapy of pulmonary arteriovenous malformations. J Vasc Interv Radiol. 2006;17(1):35-44; quiz 45.

9. Shovlin CL, Sodhi V, McCarthy A, Lasjaunias P, Jackson JE, Sheppard MN. Estimates of maternal risks of pregnancy for women with hereditary haemorrhagic telangiectasia (Osler-Weber-Rendu syndrome): suggested approach for obstetric services. BJOG. 2008;115(9):1108-1115.

10. Ahn S, Han J, Kim HK, Kim TS. Pulmonary Arteriovenous Fistula: Clinical and Histologic Spectrum of Four Cases. J Pathol Transl Med. 2016;50(5):390-393. 